\title{
Computing Gibbs free energy differences by interface pinning
}

\author{
Ulf R. Pedersen, ${ }^{1,2,{ }^{*}}$ Felix Hummel, ${ }^{2}$ Georg Kresse, ${ }^{2}$ Gerhard Kahl, ${ }^{1}$ and Christoph Dellago ${ }^{2}$ \\ ${ }^{1}$ Institute of Theoretical Physics, Vienna University of Technology, Wiedner Hauptstraße 8-10, A-1040 Vienna, Austria \\ ${ }^{2}$ Faculty of Physics, University of Vienna and Center for Computational Materials Science, Sensengasse 8/12, A-1090 Vienna, Austria
}

(Received 21 February 2013; revised manuscript received 10 June 2013; published 3 September 2013)

\begin{abstract}
We propose an approach for computing the Gibbs free energy difference between phases of a material. The method is based on the determination of the average force acting on interfaces that separate the two phases of interest. This force, which depends on the Gibbs free energy difference between the phases, is computed by applying an external harmonic field that couples to a parameter which specifies the two phases. Validated first for the Lennard-Jones model, we demonstrate the flexibility, efficiency, and practical applicability of this approach by computing the melting temperatures of sodium, magnesium, aluminum, and silicon at ambient pressure using density functional theory. Excellent agreement with experiment is found for all four elements, except for silicon, for which the melting temperature is, in agreement with previous simulations, seriously underestimated.
\end{abstract}

DOI: 10.1103/PhysRevB.88.094101

PACS number(s): 64.70.D-, 64.60.-i, 68.08.De, 71.15.Mb

An accurate location of first-order transition lines at a reasonable computational cost is of paramount importance for a wide spectrum of condensed matter systems, ranging from hard to soft materials and biological matter. Basic principles of equilibrium thermodynamics imply that for a given temperature and pressure the system resides in the phase of lowest Gibbs free energy. Phase transitions occur where Gibbs free energy differences between phases vanish, determining phase boundaries in the pressure-temperature plane. From the computational point of view, however, the task of evaluating a phase diagram represents a significant challenge, as phase transitions occur on long time scales ${ }^{1}$ such that they cannot be studied using straightforward molecular dynamics or Monte Carlo simulations.

Several numerical approaches have been proposed to cope with this problem: ${ }^{2,3}$ (i) in the indirect approach, often based on thermodynamic integration, the Gibbs free energy is computed individually for each of the phases ${ }^{4-8}$ and the coexistence line is then calculated by imposing the coexistence condition of equal Gibbs free energy. (ii) Alternatively, in the direct approach, an explicit interface is introduced between the two phases which are then simulated simultaneously in the same simulation box. At fixed pressure and temperature, the system moves towards the phase with the lower Gibbs free energy. Exactly at coexistence the thermodynamic driving force on the interface vanishes and the interface stops moving except for thermal fluctuations. Successful applications of this approach have been reported for a broad spectrum of materials. $^{9-21}$

In this contribution, we present and validate a method to compute the Gibbs free energy difference $\Delta G$ between two phases. The basic idea of this approach is to compute the average force required to pin the interface of a two-phase system via a harmonic bias potential. This external field couples to a suitably defined order parameter $Q$, which

Published by the American Physical Society under the terms of the Creative Commons Attribution 3.0 License. Further distribution of this work must maintain attribution to the author(s) and the published article's title, journal citation, and DOI. distinguishes between the phases of interest. The application of the bias potential effectively transforms the out-of-equilibrium process of the conventional moving-interface method into a well-defined equilibrium computation, in which the free energy difference $\Delta G$ is determined directly. We refer to this approach as the "interface-pinning" method. Coexistence points may subsequently be determined using Newton's rootfinding method.

To validate our approach, we have first applied it to the Lennard-Jones (LJ) model. ${ }^{22}$ Our calculations reproduce with high accuracy the solid-liquid coexistence line identified previously with other approaches. ${ }^{15,23,24}$ We have then used interface pinning in combination with $a b$ initio density functional theory to compute the melting temperatures of sodium $(\mathrm{Na})$, magnesium $(\mathrm{Mg})$, aluminum $(\mathrm{Al})$, and silicon $(\mathrm{Si})$, demonstrating that the present method is widely applicable and allows routine melting-point calculations using ab initio techniques. Traditional thermodynamic integration is difficult to combine with $a b$ initio techniques as determining the free energy of the liquid requires either (i) an integration from the ideal gas to the liquid state using the ab initio techniques, or (ii) a simple model Hamiltonian resembling the true liquid. Unfortunately method (i) fails, since most density functional theory codes yield diverging Coulomb and electronic energies, when two atoms are at the same position in space, whereas approach (ii) is suitable only when reliable model potentials exist. Such potentials are, however, not available for materials with short- and medium-range order in the liquid, such as $\mathrm{P}, \mathrm{Sb}$, chalcogenides, and binary systems (oxides, nitrides, zintl alloys), explaining the astoundingly small number of melting-point calculations using ab initio density functional theory.

Compared to the conventional direct and indirect methods used in the literature so far, interface pinning offers several advantages. In contrast to the direct approaches, interface pinning operates at well-defined equilibrium conditions, thus permitting the explicit calculation of free energy differences and interface properties. The order parameter $Q$ does not need to be a reaction coordinate capturing the entire transformation mechanism. Finally, interface pinning (IP) inherits the conceptual simplicity and general applicability of the direct approaches. The latter makes it a viable option when it is 

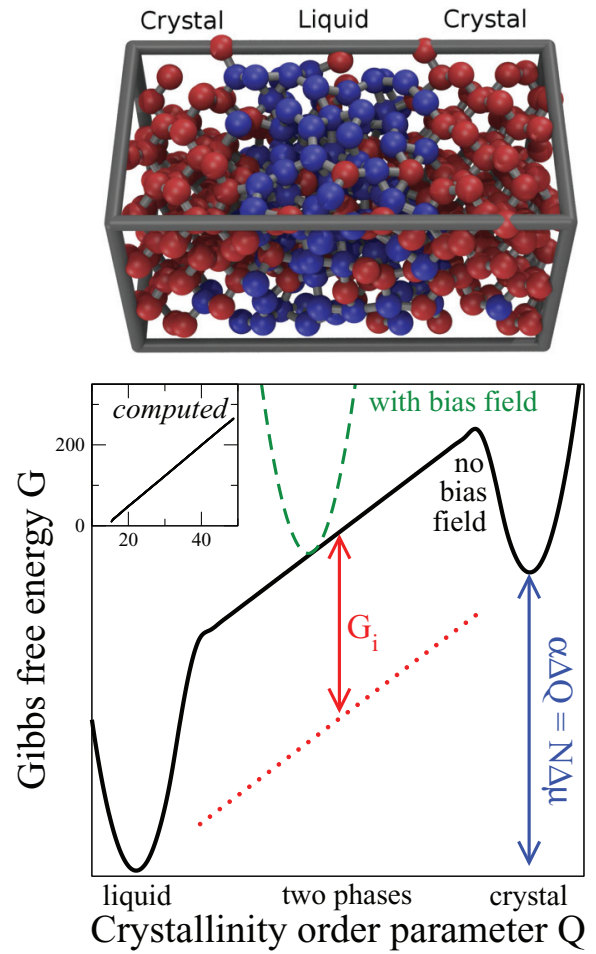

FIG. 1. (Color online) Upper panel: Crystal-liquid configuration from an ab initio simulation of $432 \mathrm{Si}$ atoms in a periodic box. Atoms are colored according to the coordination number (red indicates fourfold coordinated and blue otherwise). Lower panel: Schematic sketch of the Gibbs free energy $G(Q)$ (black solid line) as a function of the crystallinity order parameter $Q$ at a state point where the liquid is thermodynamically stable and the crystal is metastable. The double arrows indicate the interface contribution $G_{i}$ (red) and the bulk contribution $N \Delta \mu$ (blue), respectively. The dashed green curve indicates the Gibbs free energy $G^{\prime}(Q)$ with bias potential applied. The inset shows that the computed $G(Q) /\left(k_{B} T\right)$ in the two-phase region is indeed linear; here, $G(Q)$ was computed for the $\mathrm{LJ}$ model $(N=5120)$ via umbrella sampling (Refs. 2,26) using Eq. (2) with $\kappa=2$ and a range of $a$ 's.

difficult or even impossible to use thermodynamic integration. The drawback of the IP method is that interface particles are represented explicitly. This may result in larger finite-size effects and/or longer equilibration times compared to other methods. Thus, it is could be less computationally efficient than thermodynamic integration to state points of known chemical potentials.

To introduce the method, consider a two-phase crystalliquid system ${ }^{25}$ in a periodic orthorhombic box (see Fig. 1) at temperature $T$ and pressure $p$. The box lengths $X$ and $Y$ are kept constant at values for which the crystal is unstrained for a given pressure $p$, while the box length $Z$ is allowed to change in order to maintain the constant pressure $p$. Thus, the simulation is carried out under hydrostatic conditions. We refer to this ensemble as the $N p_{z} T$ ensemble. To lower the interface Gibbs free energy $G_{i}$, the system will have two interfaces in the $X Y$ plane minimizing the interface surface area. We assume that the system is large enough to represent bulk phases at least at the center of the liquid and solid slabs. Particles may then be labeled crystalline (subscript $c$ ), liquid (subscript $l$ ), or interfacial (subscript $i$ ), so that the total number of particles is $N=N_{c}+N_{l}+N_{i}$. The contributions to the total Gibbs free energy of particles in the bulk phases are determined by the chemical potentials $\mu_{c}$ and $\mu_{l}$ of the solid and liquid, respectively, and the total Gibbs free energy is $G=N_{c} \mu_{c}+N_{l} \mu_{l}+G_{i}$

When the relative distance between the interfaces changes, particles are transferred between the bulk phases. Assuming that the interface quantities $G_{i}$ and $N_{i}$ do not change when the interfaces shift due to the growth of one bulk phase at the cost of the other, the number of liquid particles may be written as $N_{l}=-N_{c}+$ const and the Gibbs free energy is given by

$$
G\left(N_{c}\right)=N_{c} \Delta \mu+\text { const, }
$$

where $\Delta \mu \equiv \mu_{c}-\mu_{l}$. Throughout the paper we will use the subscripts $c$ and $l$ for crystal and liquid properties, respectively, and let " $\Delta$ " denote "[crystal] - [liquid]."

To sample configurations in the two-phase region and to prevent the system from complete transformation into one of the pure phases, we apply a harmonic bias potential that pins the relative position of the interfaces. Let $U(\mathbf{R})$ be the energy of the unbiased system for configuration $\mathbf{R}=\left\{\mathbf{r}_{1}, \mathbf{r}_{2}, \ldots, \mathbf{r}_{N}\right\}$, and

$$
U^{\prime}(\mathbf{R})=U(\mathbf{R})+\frac{\kappa}{2}[Q(\mathbf{R})-a]^{2}
$$

the energy of the system plus the bias potential. Here, $Q(\mathbf{R})$ is a global order parameter with a linear dependence on the number of particles in the solid phase: $Q=\frac{N_{c}}{N} Q_{c}+\frac{N_{l}}{N} Q_{l}+$ $\frac{N_{i}}{N} Q_{i}$ where $Q_{c}$ and $Q_{l}$ are the average values when the system is in the solid phase and the liquid phase, respectively. Thus

$$
N_{c}=N \frac{Q}{\Delta Q}+\text { const. }
$$

[Later in the paper we introduce and discuss in detail one choice of $Q(\mathbf{R})$.] In the biased system, the position of the interfaces relative to each other will fluctuate around an average value and the order parameter $Q$ will fluctuate accordingly. The probability distribution of $Q$ is $P^{\prime}(Q)=$ $\exp \left[-G^{\prime}(Q) / k_{B} T\right] / \mathcal{Z}^{\prime}$, where $G^{\prime}(Q)$ is the Gibbs free energy along the $Q$ coordinate of the biased system, and $\mathcal{Z}^{\prime}$ is the corresponding partition function. The Gibbs free energy of the biased system may be written in terms of the unbiased free energy as $G^{\prime}(Q)=G(Q)+\frac{\kappa}{2}(Q-a)^{2}$. By insertion of Eqs. (1) and (3), it follows that $P^{\prime}(Q)$ is Gaussian,

$$
P^{\prime}(Q)=\sqrt{\frac{\kappa}{2 \pi k_{B} T}} \exp \left\{-\frac{\kappa}{2 k_{B} T}[Q-a+\alpha / \kappa]^{2}\right\},
$$

where $\alpha=N \Delta \mu / \Delta Q$ is the slope of $G(Q)$ in the two-phase region, displayed in the lower panel of Fig. 1. The distribution $P^{\prime}(Q)$ has variance $\sigma_{Q}^{2}=k_{B} T / \kappa$ and average $\langle Q\rangle^{\prime}=a-$ $\alpha / \kappa$, and the chemical potential difference between the two phases may be computed as

$$
\Delta \mu=-\kappa\left(\langle Q\rangle^{\prime}-a\right) \Delta Q / N .
$$

As a guideline, we choose $\kappa$ such that typical fluctuations in $Q$ correspond to one or a fraction of a crystal plane, and $a$ such that the system is approximately half liquid and half crystal. In practice, we find that a wide range of field parameters give the same precision of the $\Delta \mu$ estimate. $^{27}$ 
Once $\Delta \mu$ is known, coexistence points may be determined using Newton's method for finding roots. The required derivatives of $\Delta \mu$ along isobars and isotherms are given by the standard thermodynamic expressions $\partial(\Delta \mu) /\left.\partial p\right|_{T}=\Delta v$ and $\partial(\Delta \mu) /\left.\partial T\right|_{p}=-\Delta s=-(\Delta u+p \Delta v-\Delta \mu) / T$. In these relations, $\Delta v, \Delta s$, and $\Delta u$ are changes in specific volume, entropy, and energy, respectively.

To apply the interface-pinning method in practice, we must choose an order parameter $Q$ that grows linearly with the number of crystalline particles $N_{c}$ in the two-phase region. Moreover, $Q$ should be computationally inexpensive. Unlike liquids, crystals have long-ranged translational order, allowing us to use the collective density field as order parameter: $Q=\left|\rho_{\mathbf{k}}\right|$ where $\rho_{\mathbf{k}}=N^{-1 / 2} \sum_{j=1}^{N} \exp \left(-i \mathbf{k} \cdot \mathbf{r}_{j}\right)$. Here, $\mathbf{k}=$ $\left(2 \pi n_{x} / X, 2 \pi n_{y} / Y, 0\right)$ for some fixed integers $\left(n_{x}, n_{y}\right)$ that should be chosen such that $\mathbf{k}$ corresponds to a Bragg peak. This choice will maximize the contrast between the liquid and the crystal. The $z$ component of $\mathbf{k}$ is set to zero since $Z$ fluctuates in the $N p_{z} T$ ensemble. The constant $N^{-1 / 2}$ makes $Q_{l}$ system-size invariant while $Q_{c} \propto N^{1 / 2}$. Derivatives of $Q$ with respect to the particle coordinates, required to determine the forces resulting from the bias, can be computed with an algorithm scaling as $O(N)$. We note that this order parameter may be problematic in the supercooled regime, since a crystal can lower $\left|\rho_{\mathbf{k}}\right|$ by introducing long-wavelength displacements of particles. The energy penalty of such displacements is low and decreases with increasing system size. We have chosen to use $\left|\rho_{\mathbf{k}}\right|$ as order parameter for most computations, since it is generally applicable and simple. For some computations we have in addition used the Steinhardt $Q=Q_{6}$ order parameter, ${ }^{28}$ which has the advantage of being robust in the supercooled regime. The two choices of order parameter give the same $\Delta \mu$ 's within statistical error. A more detailed description of the method will be given in a forthcoming presentation. $^{27}$

To verify the method, we first used it to determine the solidliquid coexistence line of the $\mathrm{LJ}$ model with truncated pair interactions: $U(\mathbf{R})=\sum_{i>j}^{N} u\left(r_{i j}\right)$, where $u(r)=4\left(r^{-12}-\right.$ $\left.r^{-6}\right)-4\left(6^{-12}-6^{-6}\right)$ for $r<6$ and zero otherwise (LJ units are used for this model throughout the paper). Molecular dynamics (MD) simulations with a time step of $t_{\text {step }}=0.004$ were performed using the LAMMPS software package ${ }^{29}$ modified to include the bias potential. The Parrinello-Rahman barostat was used ${ }^{30}$ with a time constant of $\tau_{\mathrm{PR}}=8$ together with a Nosé-Hoover ${ }^{31,32}$ thermostat with a time constant of $\tau_{\mathrm{NH}}=4$.

Results presented in Fig. 2 demonstrate that the solid-liquid coexistence line for the LJ model computed by interface pinning agrees within high precision with data obtained using other methods. ${ }^{24,34}$ The coexistence points displayed in Fig. 2 were computed as follows. First, a crystal structure of $8 \times 8 \times 20$ face centered cubic (fcc) unit cells $(N=5120)$ was constructed and simulated at $p=1$ and $T=0.8$ for $t_{\text {sim }}=800$. All box lengths were allowed to fluctuate in order to determine the geometry of the unstrained crystal, giving $X=Y=12.85$. The unstrained crystal was then simulated for $t_{\text {sim }}=800$ in the $N p_{z} T$ ensemble, and $Q_{c}=56.31\left(n_{x}=\right.$ $\left.16, n_{y}=0\right)$ and the average partial volume $v_{c}=1.036$ was recorded. Next, a liquid was prepared by melting the crystal in a constant-volume simulation at high temperature $(T=5)$.

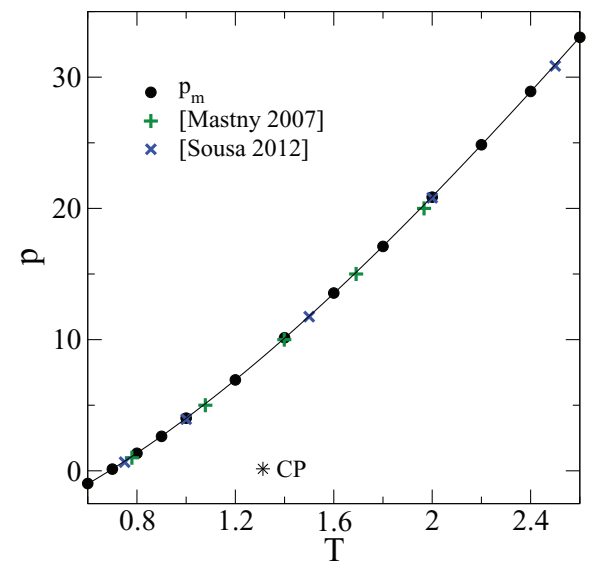

FIG. 2. (Color online) Crystal-liquid coexistence of the LJ model (filled black dots) in the ( $p, T)$ plane computed with interface pinning for the LJ model. The solid line is a cubic fit: $-0.5223 T^{3}+$ $5.017 T^{2}+5.502 T-5.989$. The computed coexistence line agrees well with results of other methods (Ref. 33): +'s and $\times$ 's are from Refs. 24 and 34, respectively. The asterisk indicates the gas-liquid critical point $\left(T_{\mathrm{CP}}=1.31 ; p_{\mathrm{CP}}=0.15\right)$ of the full LJ model (Ref. 35).

The $N p_{z} T$ ensemble (using $X=Y=12.85$ ) of the liquid was simulated for $t_{\text {sim }}=800$, and $Q_{l}=0.94$ and the average specific volume of the liquid $v_{l}=1.163$ was recorded. Then, a two-phase configuration was constructed by performing a high-temperature constant-volume simulation where particles at $z<Z / 2$ were kept at their crystal positions using harmonic springs anchored at crystal sites, with the box volume (length $Z$ ) in between that of the crystal and the liquid. The $N p_{z} T$ ensemble with the bias field of Eq. (2) with parameters $a=26$ and $\kappa=4$ was simulated for $t_{\text {sim }}=4000$ to compute $\langle Q\rangle^{\prime}=25.055$. Application of Eq. (5) yielded a chemical potential difference of $\Delta \mu=0.040$. The coexistence pressure was then determined iteratively using Newton's root-finding method along the isotherm: $p^{(i+1)}=p^{(i)}-\Delta \mu^{(i)} / \Delta v^{(i)}$, providing pressures of $p^{(i)}=\{1.0,1.320,1.337(1)\}$. In the last iteration the estimated chemical potential difference is zero within the error bar, $\Delta \mu=-0.0007$ (10) (throughout the paper numbers in parentheses indicate the statistical errors of the last digits). In Fig. 3 we confirm that $\Delta \mu(p, T)$ computed with interface pinning (symbols) is consistent with thermodynamic integration (lines).

Due to its efficiency and flexibility, the interface pinning approach can be combined with electronic structure methods and $a b$ initio molecular dynamics to computed free energy differences from first principles. For the present simulations, the method was implemented in the Vienna ab inito simulation package. ${ }^{36}$ As an example, we used interface pinning to compute the melting temperatures $T_{m}$ of the period- 3 elements $\mathrm{Na}, \mathrm{Mg}, \mathrm{Al}$, and $\mathrm{Si}$ at ambient pressure. The computed $T_{m}$ 's are shown in Table I along with simulation details. Melting temperatures were computed first for crystalline $\mathrm{Si}$ in the fourfold-coordinated cubic diamond (cd) structure (see Fig. 1). To be compatible with previous calculations, ${ }^{8,39}$ density functional theory in the local density approximation (LDA) within the framework of the projector augmented wave method was used. ${ }^{40} \mathrm{NpT}$ and $N p_{z} T$ simulations were performed using a time step of $t_{\text {step }}=3 \mathrm{fs}$ with a Parrinello-Rahman barostat ${ }^{30}$ 


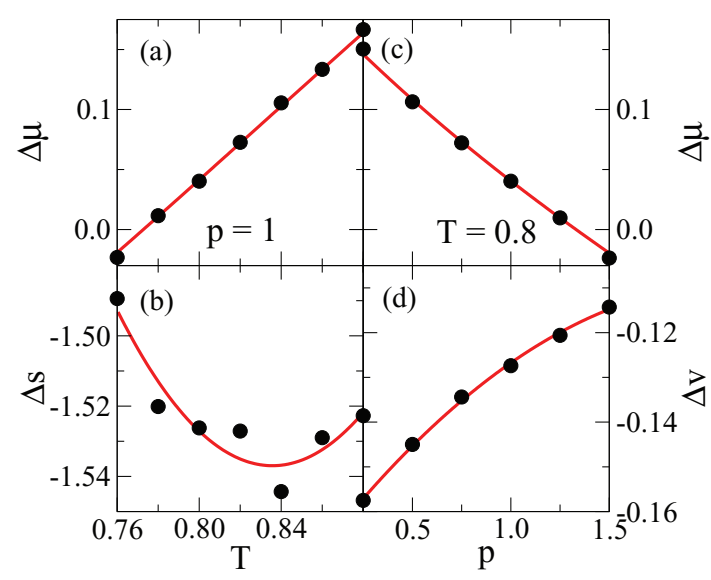

FIG. 3. (Color online) (a), (c) $\Delta \mu$ computed with interfacepinning method along an isobar and an isotherm, respectively. (b), (d) Specific entropy $\Delta s$ vs $T$ and specific volume $\Delta v$ vs $p$, respectively; the solid lines in the lower panels are quadratic polynomial fits to these data. The solid lines in the upper panels were computed by integration of these fits. The integration constants were chosen to provide the best overall agreement with the $\Delta \mu$ data.

and a Langevin thermostat. ${ }^{41}$ To compute the Si coexistence temperature at ambient pressure, we use a similar strategy as outlined for the LJ model: bulk properties of the crystal and the liquid ( $Q_{c}, Q_{l}, v_{c}, v_{l}, X$, and $Y$ ) were evaluated in simulations for $216 \mathrm{Si}$ atoms $\left(3 \times 3 \times 3\right.$ conventional cells; $\left.t_{\text {sim }}=60 \mathrm{ps}\right)$ at $T=1200 \mathrm{~K}$. Next, solid-liquid simulations with a bias field $\left(t_{\text {sim }}>30 \mathrm{ps}\right)$ were performed for four system sizes: $\{2 \times 2 \times 4,2 \times 2 \times 7,3 \times 3 \times 6,3 \times 4 \times 7\}$ conventional cubic cells corresponding to $N=\{128,224,432,672\}$ atoms. Coexistence temperatures were estimated to be $\{1189,1218,1225,1241\} \mathrm{K}$ using $T_{m} \simeq T+\frac{\Delta \mu}{\Delta s}$. Finally, finite-size effects were extrapolated assuming a $1 / N$ decay of the finite-size error yielding $T_{m}=1250(10) \mathrm{K}$. The finite-size effects are particularly large for liquid silicon, since the metallic liquid is embedded in a semiconducting host, resulting in a discretization of the electronic states in the metal (electron in a box). The present value is fully consistent with previous LDA calculations, ${ }^{8,39}$ and the discrepancy with the experimental value of $T_{m}=$ $1635 \mathrm{~K}$ originates from an underestimation of the energy difference between fourfold-coordinated semiconducting Si in the cd structure and sixfold-coordinated metallic $\mathrm{Si}$ in liquid Si resembling the $\beta$-tin structure. ${ }^{8}$ The entropy of fusion
TABLE I. Ab inito and experimental (Refs. 37 and 38) melting temperatures $T_{m}$ (in K) of period-3 elements using either $\left|\rho_{k}\right|$ or $Q_{6}$ as order parameter. "Supercell" indicates the applied supercell built from the conventional cubic cell (including liquid and solid parts). $N$ is the total particle number.

\begin{tabular}{lccccc}
\hline \hline & Unit cell & Supercell & $N$ & $Q$ & $T_{m}$ [expt.] \\
\hline $\mathrm{Na}$ & $\mathrm{bcc}$ & $5 \times 5 \times 10$ & 500 & $\left|\rho_{\mathbf{k}}\right|$ and $Q_{6}$ & $354(21)[370]$ \\
$\mathrm{Mg}$ & $\mathrm{hcp}$ & $4 \times 6 \times 8^{\mathrm{a}}$ & 767 & $\left|\rho_{\mathbf{k}}\right|$ and $Q_{6}$ & $920(20)[923]$ \\
$\mathrm{Al}$ & $\mathrm{fcc}$ & $4 \times 4 \times 8$ & 512 & $Q_{6}$ & $985(30)[933]$ \\
$\mathrm{Si}$ & $\mathrm{cd}$ & $3 \times 4 \times 7$ & 672 & $\left|\rho_{\mathbf{k}}\right|$ & $1241(20)[1635]$ \\
\hline
\end{tabular}

${ }^{\mathrm{a} B u i l t}$ from an orthorhombic four-atom $(a, \sqrt{3} a, c)$ cell.

$\Delta s\left(T_{m}\right)=3.5(1) k_{B} /$ atom and the slope of the melting curve $d T_{m} / d p=-51(7) \mathrm{K} / \mathrm{GPa}$ (computed using the ClausiusClapeyron relation) are also in agreement with previous theoretical results. $^{8,39}$ For the other elements, $\mathrm{Na}, \mathrm{Mg}$, and Al, finite-size effects are less critical, and we considered only system sizes comparable to the largest $\mathrm{Si}$ system. For these three elements, the calculations were performed using PBESOL (the Perdew-Burke-Ernzerhof functional for solids), ${ }^{42}$ which yields more accurate lattice constants than the LDA. The computed $T_{m}$ 's of $\mathrm{Na}$ and $\mathrm{Mg}$ are in excellent agreement with experimental values, while for $\mathrm{Al}$ the computed $T_{m}$ is about $6 \%$ too large (see Table I).

In summary, we have described a computational method that allows a direct evaluation of the Gibbs free energy differences between two phases at given temperature and pressure. The approach is based on the simulation of coexisting phases with a bias acting on the interface position, effectively transforming the nonequilibrium growth process into an equilibrium simulation. An appropriate choice for the dimensions of the simulation box orthogonal to the direction of interface motion ensures hydrostatic conditions. As demonstrated by computing the melting points of $\mathrm{Na}, \mathrm{Mg}, \mathrm{Al}$, and $\mathrm{Si}$ from first-principles simulations, the method is highly flexible and can be applied to systems for which conventional methods are inadequate.

This work was financially supported by the Austrian Science Fund FWF within the SFB ViCoM (F41). Computing time on the Vienna Scientific Cluster (VSC) is gratefully acknowledged.

\footnotetext{
*ulf.pedersen@tuwien.ac.at

${ }^{1}$ R. Becker and W. Döring, Ann. Phys. (Leipzig) 24, 719 (1935).

${ }^{2}$ D. Frenkel and B. Smit, Understanding Molecular Simulation: From Algorithms to Applications, 2nd ed., Computational Science Series Vol. 1 (Academic Press, London, 2002).

${ }^{3}$ C. Vega, E. Sanz, J. L. F. Abascal, and E. G. Noya, J. Phys.: Condens. Matter 20, 153101 (2008).

${ }^{4}$ B. Widom, J. Chem. Phys. 39, 2808 (1963).

${ }^{5}$ W. G. Hoover and F. H. Reee, J. Chem. Phys. 49, 3609 (1968).

${ }^{6}$ W. G. Hoover, S. G. Gray, and K. W. Johnson, J. Chem. Phys. 55, 1228 (1971)

${ }^{7}$ G. E. Norman and V. S. Filinov, High Temp. 7, 216 (1969).
}

${ }^{8}$ D. Alfè and M. J. Gillan, Phys. Rev. B 68, 205212 (2003).

${ }^{9}$ A. J. C. Ladd and L. V. Woodcock, Chem. Phys. Lett. 51, 155 (1977).

${ }^{10}$ J. Q. Broughton and G. H. Gilmer, J. Chem. Phys. 84, 5749 (1986).

${ }^{11}$ U. Landman, W. D. Luedtke, R. N. Barnett, C. L. Cleveland, M. W. Ribarsky, E. Arnold, S. Ramesh, H. Baumgart, A. Martinez, and B. Khan, Phys. Rev. Lett. 56, 155 (1986).

${ }^{12}$ A. Mori, R. Manabe, and K. Nishioka, Phys. Rev. E 51, R3831 (1995).

${ }^{13}$ A. Kyrlidis and R. A. Brown, Phys. Rev. E 51, 5832 (1995).

${ }^{14}$ P. M. Agrawal, B. M. Rice, and D. L. Thompson, J. Chem. Phys. 119, 9617 (2003). 
${ }^{15}$ J. R. Morris and X. Song, J. Chem. Phys. 116, 9352 (2002).

${ }^{16}$ J. J. Hoyt and M. Asta, Phys. Rev. B 65, 214106 (2002).

${ }^{17}$ R. Sibug-Aga and B. B. Laird, J. Chem. Phys. 116, 3410 (2002).

${ }^{18}$ R. G. Fernández, J. L. Abascal, and C. Vega, J. Chem. Phys. 124, 144506 (2006).

${ }^{19}$ N. S. Weingarten, W. D. Mattson, and B. M. Rice, J. Appl. Phys. 106, 063524 (2009).

${ }^{20}$ T. Zykova-Timan, J. Horbach, and K. Binder, J. Chem. Phys. 133, 014705 (2010).

${ }^{21}$ U. R. Pedersen and P. Harrowell, J. Phys. Chem. B 115, 14205 (2011).

${ }^{22}$ J. E. Lennard-Jones, Proc. R. Soc. London, Ser. A 106, 463 (1924).

${ }^{23}$ J.-P. Hansen and L. Verlet, Phys. Rev. 184, 151 (1969).

${ }^{24}$ E. A. Mastny and J. J. de Pablo, J. Chem. Phys. 127, 104504 (2007).

${ }^{25}$ D. P. Woodruff, The Solid-Liquid Interface (Cambridge University Press, Cambridge, 1973).

${ }^{26}$ M. R. Shirts and J. D. Chodera, J. Chem. Phys. 129, 124105 (2008).

${ }^{27}$ U. R. Pedersen J. Chem. Phys., doi:10.1063/1.4818747.

${ }^{28}$ P. J. Steinhardt, D. R. Nelson, and M. Ronchetti, Phys. Rev. B 28 , 784 (1983).

${ }^{29}$ S. J. Plimpton, J. Comput. Phys. 117, 1 (1995); http://lammps. sandia.gov

${ }^{30}$ M. Parrinello and A. Rahman, J. Appl. Phys. 52, 7182 (1981).
${ }^{31}$ S. Nosé, J. Chem. Phys. 81, 511 (1984).

${ }^{32}$ W. G. Hoover, Phys. Rev. A 31, 1695 (1985).

${ }^{33}$ We notice small systematic deviations of $\Delta p_{m}<0.1$ related to truncation of LJ interactions.

${ }^{34}$ J. M. G. Sousa, A. L. Ferreira, and M. A. Barroso, J. Chem. Phys. 136, 174502 (2012).

${ }^{35}$ J. J. Potoff and A. Z. Panagiotopoulos, J. Chem. Phys. 109, 10914 (1998).

${ }^{36}$ G. Kresse and J. Furthmüller, Phys. Rev. B 54, 11169 (1996).

${ }^{37}$ R. Hultgren, P. D. Desai, D. T. Hawkins, M. Gleiser, K. K. Kelly, and D. D. Wagman, Selected Values of the Thermodynamic Properties of the Elements (American Society for Metals, Materials Park, OH, 1973), p. 294.

${ }^{38}$ M. W. Chase, J. L. Curnutt, J. R. Downey, R. A. McDonald, A. N. Syverud, and E. A. Valenzuela, J. Phys. Chem. Ref. Data 11, 695 (1982).

${ }^{39}$ O. Sugino and R. Car, Phys. Rev. Lett. 74, 1823 (1995).

${ }^{40}$ P. E. Blöchl, Phys. Rev. B 50, 17953 (1994).

${ }^{41}$ P. Langevin, C. R. Hebd. Seances Acad. Sci. 146, 530 (1908).

${ }^{42}$ J. P. Perdew, A. Ruzsinszky, G. I. Csonka, O. A. Vydrov, G. E. Scuseria, L. A. Constantin, X. Zhou, and K. Burke, Phys. Rev. Lett. 100, 136406 (2008). 